\title{
The effect of tempo and tone duration on rhythm discrimination
}

\author{
STEPHEN HANDEL \\ University of Tennessee, Knoxville, Tennessee
}

\begin{abstract}
Rhythm constancy was investigated in two experiments. In Experiment 1, the first rhythm was presented at one tempo, the second rhythm was presented at a different tempo, and subjects judged whether the relative timing structures were identical (i.e., was the first $r$ hythm merely sped up or slowed down to generate the second rhythm?). For the nonmetric rhythms used here, subjects perceived the rhythm in terms of the figural grouping of the tones, and rhythm constancy broke down between slower and faster tempos. In Experiment 2, the first rhythm was presented in tones of one duration; the second rhythm was presented in tones of a different duration; and subjects judged whether the timing structures of the tone onsets were identical (the two rhythms were presented at the same tempo). These results indicated a high degree of constancy; subjects found it easy to discriminate the timing structures. These results confirm that the onset timing is critical to rhythm perception and suggest that rhythm perception at slower rates (2 elements/sec) differs from rhythm perception at faster rates (3-4 elements/sec).
\end{abstract}

Perceptual constancy continues to provide a challenge to theoretical orientations. The abstraction of visual or auditory objects in spite of diverse orientations, sizes, octaves, timbres, and tempos led Ehrenfels in 1890 to postulate the attribute of form for melodies and eventually led the Gestalt psychologists to argue that form was primary. Historically, the classic problems of size and shape constancy of visual objects have generated the vast majority of the research, but this is probably due to the ease of experimentation. Equally important issues need to be addressed in the auditory domain, ranging from simple octave melodic transformations to more complex harmonic and timing changes (see, e.g., Dowling, 1986). The purpose of this paper is to consider two aspects of perceptual constancy of rhythmic patterns. First, can listeners perceive relative timing structures between the onsets of tonal elements when a rhythm is played at different tempos? Second, can listeners perceive relative timing structures when the tempo is identical, but the tonal elements have different durations?

It is usually assumed that temporal patterns can be slowed down or sped up without changing the perceived rhythm. Although each interval between the onsets of successive elements is changed, the ratio between the intervals is invariant. Thus, it appears likely that identical accents, groupings, and metric structures would be perceived. Nonetheless, several kinds of outcomes suggest that changes in tempo do in fact alter the nature of auditory perception in general, and that of rhythm perception in specific.

I thank Patrick Gaffney for his help in running the experiment, and Mari Reiss Jones for her incisive comments on the manuscript. Correspondence should be addressed to S. Handel, Department of Psychology, University of Tennessee, Knoxville, TN 37996-0900.
Garner (1974) and co-workers presented repeating auditory binary patterns that were composed of two frequencies (e.g., xxxoxxoxxxxoxxox). Subjects listened to the sequence, and, when ready, described the pattern in terms of the $x, o$ sequence. At faster presentation rates ( 2 elements/sec or faster), the patterns were perceived by listeners with little sense of trying to figure them out; each pattern was perceived as a whole. The pattern was heard as a series of coherent runs of identical elements. Thus, the presentation sequence xxxoxxoxxxxoxxox would be described as the repeating pattern xxxxoxxo to create the initial run of four elements. The first element of the run would often be heard as accented. At slower rates, the patterns tended to be learned and verbally encoded with active hypothesis testing. In this case, the initial elements of the sequence determined the description (i.e., xxxoxxoxxxxoxxox would be described as xxxoxxox). At these slower rates, there was no sense of stronger or weaker beat elements.

Handel and Lawson (1983) presented polyrhythms (defined as the simultaneous presentation of two or more isochronous rhythms that do not have a common denominator, such as $3 \times 4,2 \times 5 \times 7$ ) at different presentation rates, and subjects were required to tap out the meter of the polyrhythms. The most important factor determining which rhythm became the meter was the onset-to-onset interval between elements within one isochronous line. If the onset-to-onset interval between elements within one line is greater than $800 \mathrm{msec}$, the elements appear unconnected and the rhythm line is thus unsuited to serve as the meter. Alternatively, if the interval between elements within one line is less than $200 \mathrm{msec}$, the elements appear grouped with subjectively accented and unaccented elements. Here too, the rhythmic line is unsuited to serve as the meter to provide a regular beat. Thus, the perceived meter changed as a function of the overall tempo. 
Monahan and Hirsh (1990) required subjects to discriminate between six-tone metric thythmic patterns in which the onset of one tone could be delayed. There were large interactions in the difference threshold due to the tempo, rhythmic pattern, and position of the delayed tone, suggesting that different discrimination models were required at different tempos.

Ten Hoopen (1992) summarized several experiments on the detection of temporal deviations in isochronous patterns. Ten Hoopen argued that the difference threshold is relatively constant for intervals less than $250-300 \mathrm{msec}$ and that Weber's law holds for intervals greater than $300 \mathrm{msec}$. On this basis, he concluded that temporal processing at rates greater than three tones/sec is fundamentally different from temporal processing at rates less than three tones/sec. At the faster rates, discrimination is based on a primitive, global template matching operation, because listeners are unable to build up timed anticipations. Moreover, Ten Hoopen found that the perceived ratio between successive intervals was compressed at faster rates, although it was accurate at slower rates. Consider a ratio of 2:3 (e.g., in a sequence of three tones, the first interval is $200 \mathrm{msec}$ and the second is $300 \mathrm{msec}$ ). If the two intervals are, physically, $80 \mathrm{msec}$ and $120 \mathrm{msec}$, listeners perceive the second interval to be equal to $90 \mathrm{msec}$. However, if the physical length of the intervals is lengthened to $240 \mathrm{msec}$ and $360 \mathrm{msec}$, listeners perceive the second interval correctly, at approximately $360 \mathrm{msec}$. Thus, these results also suggest a limitation to rhythm constancy. As the tempo changes, the perceived ratios between pairs of equivalent intervals will not be equal, which can lead listeners to perceive different rhythms at slower and faster tempos.

Handel (1992) investigated the differentiation and discrimination of rhythmic structure of nonmetric rhythms identical to the ones used in the present experiment. These rhythms were presented at a slower (e.g., 1.9 tones/sec) and a faster $(3.6$ tones/sec) presentation rate. Two outcomes suggested that these rhythms were perceived differently at the two rates. First, the best discrimination between two rhythms at the slow rate occurred when the two rhythms were alternately presented (e.g., abababab), but the best performance at the faster rate occurred when the rhythms were cycled twice and then alternated (e.g., aabbaabb). Second, the difficulty in discriminating between two rhythms varied between the rates. At the slow rate, it was easier to discriminate between two rhythms that differed in the timing of the initial elements, but at the fast rate, it was easier to discriminate between two rhythms that differed in the timing of the final elements.

Sorkin and Montgomery (1991) investigated the effect of tempo change on the ability to distinguish whether two arrhythmical tonal sequences repeated once were the same or different. The results indicated that performance dropped nearly to chance if the tempo of the second sequence was increased by a factor of 1.7 and that performance dropped moderately if the tempo of the second sequence was decreased by a factor of 1.4. Sorkin and Montgomery suggest that the performance decrement was due either to an internal noise component interfering with the discrimination of intervals that was proportional to the magnitude of the difference in tempo or to different attentional strategies used by subjects at slower and faster tempos. (See Monahan \& Hirsh, 1990, and Handel, 1992, discussed above.)

All these results indicate that rhythmic organization changes as a function of tempo. The reasons for these changes are not understood. There seems to be a consensus that at slower tempos listeners build up a set of timing expectations based on the initial elements, but that at faster tempos, listeners evolve a wholistic template in which the final elements predominate. These differences may be due to simple sensory discrimination limitations or to primitive grouping schemas (see Bregman, 1990) that function at different presentation rates. Whatever the reason, tempo affects both element grouping and the meter and accent formation involved in rhythmic organization (see Lerdahl \& Jackendoff, 1983).

It has also been assumed that rhythm is based on the onset-to-onset timing of the elements, so that the duration of elements is relatively unimportant. Thus, in a tone sequence in which the onsets are separated by $100 \mathrm{msec}$, each note could be $25 \mathrm{msec}, 50 \mathrm{msec}$, or $75 \mathrm{msec}$ and not affect the perceived rhythm. Lerdahl and Jackendoff (1983) explicitly make this assumption by using the concept of "attack points" to define grouping and meter preference rules. Furthermore, Yeston (1976) argues that, by itself, the attack-point sequence will always establish the extreme rhythmic foreground. The emergence of a metric structure depends on the consonance of several hierarchical levels, such that the attack points or beats at higher levels occur at integer multiples of the lower (or surface) levels. The fact that the attack points at different levels are synchronous, even though the durational values at each level may differ, has led music theory to minimize the rhythm structuring effect due to note duration within an attack-point sequence. The experimental evidence concerning duration is sparse.

The most relevent research was done by Vos and Ellerman (1989). They presented sequences of tones with fixed onset-to-onset intervals of $1,000 \mathrm{msec}$ and varied the tone/silent offset-to-onset interval durations from 100 msec tone $/ 900-\mathrm{msec}$ interval to $900-\mathrm{msec}$ tone $/ 100-\mathrm{msec}$ interval. They found no difference in the accuracy of reproduction across the nine steps. Vos and Ellerman (1989) argue that performers pay more attention to onsetto-onset timing (they use the term interattack timing) and that at slow or fast tempos, the silent intervals (i.e., the rests) rather than the tone durations are likely to be misproduced. In related work, Handel (1974), Handel and Lawson (1983), and Hopkins (1992) investigated whether differences in tone duration were a determinant of rhythmic organization. They found that although short staccato elements appear to create a better sense of meter than longer ones do, durational differences provide only a weak basis for rhythmic organization. 
In sum, the effect of element duration is not strong. These results therefore reinforce the prevailing theoretical view that the timing of the onset of tones creates the sense of rhythm.

\section{GENERAL METHOD}

To study the effect of tempo and duration on rhythmic organization, we made use of a variant of the same-different task used by Handel (1992). In the original experiment, the first rhythm was presented in one pitch and then the second rhythm was presented in another pitch at the same tempo and element duration. The subject's task was to judge whether the timings between elements of the two rhythms were the same or different. In the present research, the first rhythm was presented in an identical fashion, but the second rhythm was presented in another pitch at a slower or faster tempo with the same tone durations (Experiment 1) or the second rhythm was presented in another pitch at the same tempo with a shorter or longer tone duration (Experiment 2). In both experiments, the subjects judged whether the timing structures were the same or different. By comparing discrimination in cases in which the tempo or element duration differed and discrimination in cases in which tempo and duration were constant, it is possible to determine the ways in which tempo and duration affect rhythmic discrimination and organization.

To demonstrate the conditions concretely, consider the four possible kinds of conditions involving tempo change. Imagine two simple rhythms composed of 11 units; each unit may be a tone or a silent interval. Rhythm 1 is $x . x . . x . x . .$. , and Rhythm 2 is $x . x . x . . x \ldots$ (the $x$ s represent tone elements; the dots represent isochronous equal-duration silent time intervals). If each unit is $\mathbf{5 0} \mathrm{msec}$, the length of each rhythm is $550 \mathrm{msec}$, at which time the initial element would recur if the rhythms were recycled. Now consider the same rhythms played at one half the tempo, $x \ldots x \ldots . . . . . x \ldots \ldots$ and $x \ldots x \ldots x \ldots . . . . \ldots \ldots$, so that the length of each pattern is now 1,100 msec.

In a single trial, the first rhythm (a) was presented twice, in one pitch $(x)$, the second rhythm was presented twice in a different pitch $(y)$, and this sequence was then repeated without interruption. The second rhythm was either identical to the first (with the exception of the different pitch) or a different one (again with a different pitch). Thus, a trial was either $\mathrm{a}(\mathrm{x}) \mathrm{a}(\mathrm{x}) \mathrm{a}(\mathrm{y}) \mathrm{a}(\mathrm{y}) \mathrm{a}(\mathrm{x}) \mathrm{a}(\mathrm{x}) \mathrm{a}(\mathrm{y}) \mathrm{a}(\mathrm{y})$, where $\mathrm{a}(\mathrm{x})$ stands for rhythm a with pitch $x$, if the two thythms were identi$c a l$, or $a(x) a(x) b(y) b(y) a(x) a(x) b(y) b(y)$, where $b(y)$ stands for rhythm $b$ with pitch $y$, if the two rhythms were different.

The four conditions were generated by crossing the two rhythm conditions (same rhythms vs. different thythms) with the two tempo conditions (same tempos vs. different tempos). The four types of condition are illustrated below:

In the first, the two rhythms were identical (Rhythm 1 above) and were played at the same tempo (only half of the trial is shown to conserve space; $x$ and $y$ represent the different frequencies:

$$
x . x . . x . x \ldots x . x . . x . x \ldots y . y, y . y \ldots y \cdot y, y, y \ldots \text { (fast rate) }
$$

In the second, the two rhythms were different (Rhythms 1 and 2 above) and were played at the same tempo:

$$
\text { x.x..x.x...x.x..x.x...y.y.y..y...y.y.y..y... }
$$

In the third. the two rhythms were identical but were played at different tempos (fast-slow):

$$
x . x \ldots x . x \ldots x . x \ldots x . x \ldots y \ldots y \ldots y \ldots y \ldots \ldots y \ldots y \ldots y \ldots y
$$

In the fourth, the two rhythms were different and were played at different tempos (slow-fast):

$$
x \ldots x \ldots \ldots \ldots x \ldots \ldots x \ldots x \ldots \ldots \ldots x \ldots \ldots y, y, y \ldots y \ldots y \cdot y \cdot y \ldots y \ldots
$$

The same four kinds of conditions would occur for element duration: identical patterns-identical durations; different patterns-identical durations; identical patterns-different durations; different patterns-different durations.

To study the effects of tempo and duration in clearest form, we used nonmetric patterns based on unfilled intervals. Meter is the sense of a regular periodic sequence of subjectively stronger and weaker beats that characterize music. The meter forms a time-based lattice that serves to create the rhythmic organization. The meter occurs at several hierarchical levels at once, so that the beats at higher levels occur at integer multiples of the beats at the lower levels. The strength of any beat is determined by the number of levels at which the beat appears. For example, suppose we have a rhythm based on a repeating unit of 16 elements. The strong beats would occur at Elements 1, 5, 9, 13; stronger beats would occur at Elements 1 and 9; and the strongest beat would occur at Element 1 . Notes that fall at the points of the strong beats become accented and notes that fall at the points of weak beats are unaccented.

To define metric and nonmetric rhythms, we will make use of the rules suggested by Povel and Essens (1985) for sequences of identical elements separated by different lengths of silent intervals. One rule is most critical: A tone should occur at the positions of the strong meter beats, and a silence or rest should not. For rhythms 16 units long, we will operationally define the metric strength of a rhythm by the occurrence of tones at the beat positions $1,5,9$, and 13 . Thus, the rhythm $x . x . x . . . x \ldots x . .$. would be metric, because tones fall on the stronger beats at Positions 1, 5, 9, and 13, but the rhythm $x . x \ldots x . . x . x \ldots$ would be nonmetric, because tones do not fall at Positions 5, 9, or 13. From this perspective, rhythms are not simply metric or nonmetric. Instead, each rhythm is metric to some degree, depending on the strength of the metric interpretation it evokes. Povel and Essens argue that listeners attempt to find a meter to fit a rhythmic pattern and that highly metric patterns more easily induce an internal clock that codes the rhythm temporally. Thus, these rhythms would be easier to represent and reproduce.

We chose to investigate the perception of nonmetric rhythms here and in previous work (Handel, 1992) because we wanted to understand the perception of the timing structure without the use of an intervening preexisting schema (Palmer \& Krumhansl, 1990). It appears that nonmetric rhythms are organized into bounded groups of elements that follow one another, although the timings between the onsets of the successive groups are not encoded or compared (Handel, 1992; Povel \& Essens, 1985). Bamberger (1978) and Povel and Essens term this figural grouping, because the runs of elements are figures perceived against an ordinal ongoing time and not perceived against a temporal grid in which every element can be timed. For example, the nonmetrical pattern illustrated above, $x . x \ldots x . . x . x \ldots$, would be coded 2 , silence, 1 , silence, 2 , silence, and the lengths of the silent intervals would be coded only roughly, if at all. We will write this grouping as $2-1-2$. Thus, we might expect that the similar nonmetric pattern $x . x . . x \ldots x . x \ldots$ would be coded identically as 2 , silence, 1 , silence, 2 , silence $(2-1-2)$ and therefore would be easily confused with the former pattern.

To summarize at this point: Two experiments involved the discrimination between two nonmetric rhythmic patterns. In Experiment 1 , the presentation rate or tempo of the two rhythms was varied to determine whether there would be rhythmic constancy. That is, if the same thythm was played at two different rates, could listeners pick up that the relative timings between elements underlying the rhythmic structures were identical? Conversely, if two different rhythms were played at different rates, could listeners pick up that the relative timings were discrepant? Previous research, reviewed above, suggested that this should have been a difficult task, because rhythmic organization seems to differ at slower and faster presentation rates. In Experiment 2, the duration of the tones in each rhythm was varied (the durations of all tones within one rhythm 
would be identical) to determine whether there would be rhythmic constancy. That is, if the same rhythm defined in terms of onsetto-onset intervals was played with different tone durations (i.e. staccato vs. legato in musical terms), could listeners pick up the identical rhythmic structure? Results of previous research had suggested that this should have been an easy task, because tone duration per se appears to play only a minor role in rhythmic organization.

\section{EXPERIMENT 1}

The general strategy for the experimental conditions was to present two rhythms at different rates and require subjects to judge whether the two were the same or different. In baseline conditions, the two rhythms were presented at the same rate. The comparison between experimental and baseline conditions can determine the effect of tempo on rhythmic discrimination.

\section{Method}

Subjects. All 123 subjects were undergraduate volunteers at the University of Tennessee who received course credit for their participation. The subjects were run in small groups of 1-3.

Rhythms. All rhythms, shown in Table 1, were based on five tones embedded in a repeating pattern of 16 units. The rules used to construct the patterns were as follows: (1) a tone always occurred at the Ist unit; (2) a tone always occurred at the 12th unit, and no tone occurred at Units $13,14,15$, and 16; (3) there was at least 1 silent unit, but there were no more than 3 silent units between the tones in any pair. The combination of Rules 1, 2, and 3 made the longest silent interval (4 units long) occur at the end of the rhythm, so that each rhythm would naturally be organized ending at that interval according to the gap principle proposed by Garner and others (Garner, 1974; Handel, 1974). Thus the preferred organization of each rhythm began at the 1st unit, as is displayed in Table 1. The rhythms are nonmetric in two ways. First, no rhythm has tones that occur on all the stronger beat elements $1,5,9$, and 13 (the strength of the beats is indicated by the number of asterisks in Table 1). One rhythm (No. 12) has tones at three of the four stronger beat elements: 1, 5, and 9. Seven thythms (Nos. 2, 3,5, $6,7,10$, and 13) have tones only at the strongest beat at the beginning of the rhythm. Second, the onset-to-onset intervals occur in 2:3:4 ratios, so that the ratio between intervals is a simple integer in only one instance. For every trial, all thythms started at the 1st unit and ended at the 16th unit.

The preceding rules generated 16 possible patterns, 13 of which were used in the experiment. Three patterns, all beginning with a tone in Unit 1 followed by three silent units, were eliminated merely to limit the number of possible rhythms. A total of 33 pairs of rhythms were constructed. Thirteen pairs consisted of two identical rhythms, I pair for each of the rhythms. Twenty pairs contained two different rhythms. The pairs were selected from the 78 possible pairs to create a variety of differences. Specifically, the two rhythms in 3 pairs had the identical figural organization (Pairs 14-16 in Table 1, which represent all the possible ones); the two rhythms in 11 pairs differed in the location of one tone (e.g., the fourth tone in Pair 21); and the two rhythms in 6 pairs differed in the location of two or three tones (e.g., the third and fourth tones in Pair 17). To use an alternative categorization: in 6 pairs, the first difference occurred at the second tone (e.g., Pair 19); in 9 pairs, the first difference occurred at the third tone (e.g., Pair 18); and in 5 pairs, the first difference occurred at the fourth tone (e.g., Pair 24). The majority, 18 out of the 20 pairs, were used by Handel (1992) and were selected to ensure that some pairs of rhythms were very difficult to distinguish whereas other pairs were very easy to distinguish. Across the 20 different pairs, each rhythm was used three or more times (except for Nos. 7, 9, and 13, which were used twice, twice, and once, respectively), and each rhythm occurred equally often as the first and second rhythm of the pair. Although the rhythms were always presented in the same order here. comparisons in Experiment 2 did not show any effect of the order of the two rhythms.

Task. On every trial, two rhythms were presented and the subject judged whether the rhythms were the same or different in terms of the relative timing among the elements. The subjects used a fourpoint rating scale in which 1 represented very sure identical, 2 represented fairly sure identical, 3 represented fairly sure different, and 4 represented very sure different. The first rhythm was al ways presented with a higher pitch tone $(586 \mathrm{~Hz}$, triangle waveform) and the second rhythm was always presented with a lower pitch tone $(440 \mathrm{~Hz}$, triangle waveform).

Presentation rates. The rhythms were presented at three different rates. At the slow rate, the onset-to-onset interval between adjacent units was $167 \mathrm{msec}$, so that the onset-to-onset interval between tones in the sequence $x . . x$ was $668 \mathrm{msec}$. At this rate, the length of one repetition was $2.67 \mathrm{sec}$ and there were 1.9 elements $/ \mathrm{sec}$. At the medium rate, the interval was $133 \mathrm{msec}$, so that the length of one repetition was $2.12 \mathrm{sec}$ and there were 2.3 elements $/ \mathrm{sec}$. At the fast rate, the interval was $88 \mathrm{msec}$, so that the length of one repetition was $1.4 \mathrm{sec}$ and there were 3.6 elements $/ \mathrm{sec}$. At all presentation rates, each tone was composed of a $10-\mathrm{msec}$ onset ramp, a 30-msec steady state, and a 10-msec offset ramp.

The rhythms were generated by using BRS-Foringer modules. The rhythms were prerecorded and presented to subjects on cassette tapes. The experimental session took place in a small room $(3 \times 4 \mathrm{~m})$ with acoustical ceiling tile. The subjects were seated $2.5 \mathrm{~m}$ from two vertically stacked speakers, each of which presented the entire rhythm. The rhythms were presented at a comfortable listening level, approximately $65 \mathrm{~dB}$ SPL (subjects were allowed to adjust the loudness if they wished).

Tempo conditions. For all conditions, the first rhythm was repeated twice; then, without a pause, the second was repeated twice; and, again without a pause, this sequence was recycled. This condition was selected because it was one of the easiest conditions in Handel (1992). Preliminary work had indicated that discriminating rhythms at different rates was very difficult, so that it was thought that the multiple comparisons available in the double alternation would aid performance.

There were nine conditions. In three, the first and second rhythms were presented at the same rate: slow, medium, and fast. In six, the first and second rhythms were presented at different rates: (1) slow-medium; (2) medium-slow; (3) medium-fast; (4) fastmedium; (5) slow-fast; and (6) fast-slow. The notation for the slow-fast condition indicates that the first pattern was presented at the slow rate; the second pattern, at the fast rate.

Experimental design. The nine conditions were broken into 12 blocks of three conditions each. The first condition of each block always had the same rate, but the next two conditions had different rates. For example, in 4 blocks the slow-rate condition was combined with the medium-fast and slow-fast conditions, so that the four blocks counterbalanced the different tempo conditions in terms of the direction (e.g., slow-fast as opposed to fast-slow) and order of presentation: (1) slow, medium-fast, fast-slow; (2) slow, fastmedium, slow-fast; (3) slow, fast-slow, medium-fast; and (4) slow, slow-fast, fast-medium. The analogous procedures were used for the medium- and fast-rate conditions to create four blocks apiece; the medium-rate condition was combined with the slow-fast and medium-slow conditions and the fast-rate condition was combined with the slow-medium and fast-slow conditions. The method of blocking was used to increase the number of times the slow-fast and fast-slow conditions occurred, because these conditions maximized the tempo difference. Across the 12 blocks, each same-rate condition occurred four times; the slow-fast and fast-slow conditions occurred six times apiece; the slow-medium and fast-medium conditions occurred four times apiece; and the medium-slow and medium-fast conditions occurred two times apiece. 
Table 1

Average Ratings (AR) and Percent Correct (PC) for All Pairs of Rhythms at All Tempo Conditions

\begin{tabular}{|c|c|c|c|c|c|c|c|c|c|c|c|}
\hline \multirow{3}{*}{$\begin{array}{c}\text { Pair } \\
\text { Number }\end{array}$} & \multirow[b]{3}{*}{ Numbert } & \multirow{3}{*}{$\begin{array}{l}\text { Rhythm } \\
\text { Timing }\end{array}$} & \multirow{3}{*}{$\begin{array}{l}\text { Figural } \\
\text { Grouping }\end{array}$} & \multicolumn{8}{|c|}{ Tempo Conditions } \\
\hline & & & & \multicolumn{2}{|c|}{ Same } & \multicolumn{2}{|c|}{$\begin{array}{c}\text { Slow/ } \\
\text { Medium }\end{array}$} & \multicolumn{2}{|c|}{$\begin{array}{c}\text { Medium/ } \\
\text { Fast }\end{array}$} & \multicolumn{2}{|c|}{$\begin{array}{c}\text { Slow/ } \\
\text { Fast }\end{array}$} \\
\hline & & & & AR & PC & AR & PC & AR & PC & AR & PC \\
\hline
\end{tabular}

Identical-Rhythm Pairs

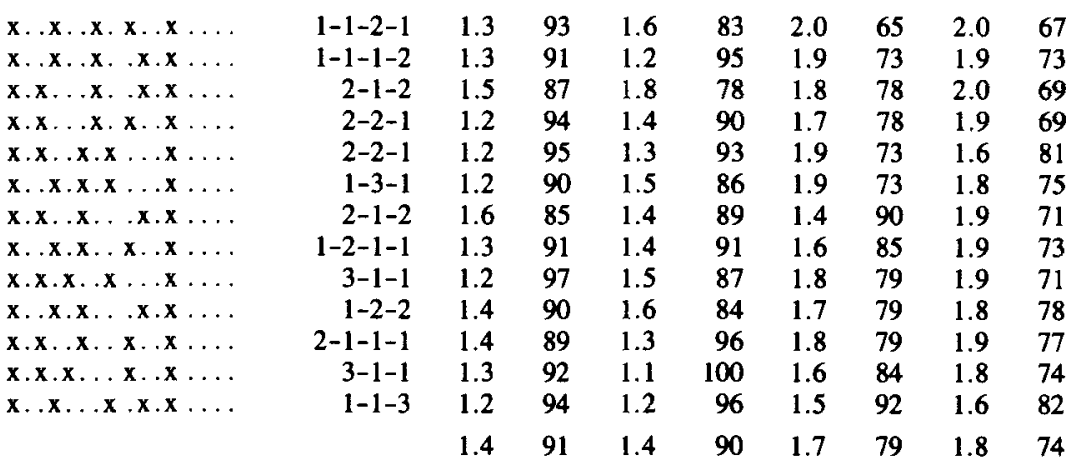

Mean

Different-Rhythm Pairs

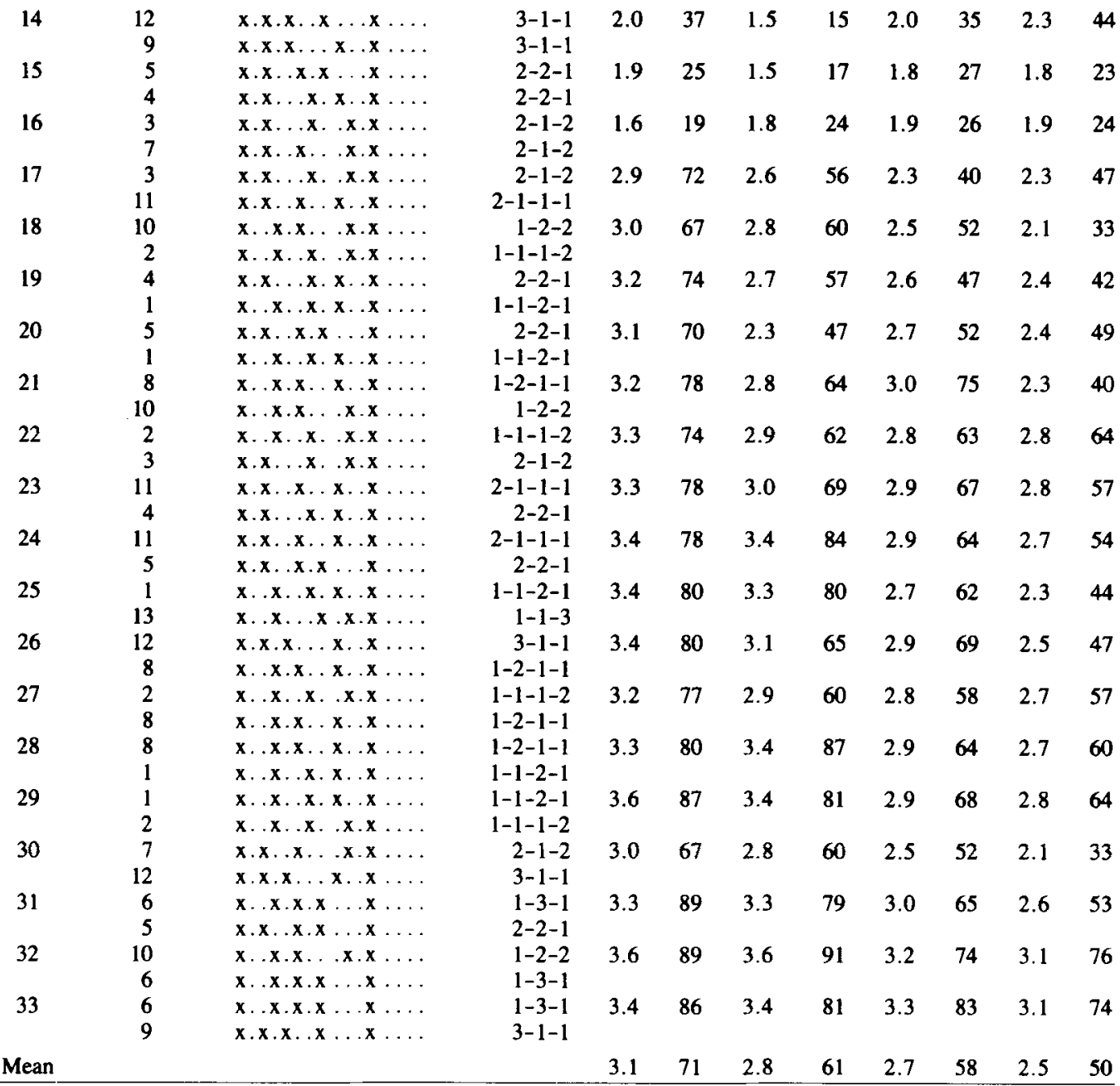

$†$ The first rhythm of each pair is the top one. $¥$ The asterisks within the table indicate location of strong and weak beats. 
Each subject was presented one block of three conditions. Each condition consisted of 33 rhythm pairs -13 identical pairs and 20 different pairs-presented in random order. Two strategies were used to acquaint the subject with each condition. First, before the actual presentation, four practice trials with simpler four-tone rhythms in the same conditions were given. Two of the trials consisted of identical pairs, and two of the trials consisted of different pairs. If a subject was confused, these patterns were repeated until the subjects felt confident. Second, the first two rhythm pairs were repeated later among the 33 experimental trials (thus, there were 35 trials per condition). The subjects were not told that these were practice trials, and the results were not scored. The subjects did not receive any feedback about their performance. There were shor breaks between the three conditions, so that the experimental session lasted $60 \mathrm{~min}$.

The numbers of subjects for the conditions were as follows: slow, $n=31$; medium, $n=34$; fast, $n=35$; slow-medium, $n=29$; medium-slow, $n=26$; medium-fast, $n=25$; fast-medium, $n=32$; slow-fast, $n=57$; fast-slow, $n=49$. The differing numbers of subjects reflect the variation in the number of subjects at the experimental sessions and the fact that not all the subjects were able to complete the last condition in a block, because of time constraints.

\section{Results}

The data were summarized with the use of two measures. The first was the average rating of each rhythm pair. Better performance for the identical pairs would result in average ratings of between 1 and 2 ; better performance for the different pairs would result in average ratings of between 3 and 4 . The second measure was the percentage correct obtained by combining the 1 rating (very sure identical) and 2 rating (fairly sure identical) for the identical pairs and combining the 3 rating (fairly sure different) and 4 rating (very sure different) for the different pairs. Although these measures are obviously correlated, each can provide slightly different views of the outcomes.

Preliminary analysis. The first step was to determine whether the order of presentation affected discrimination. For every subject, the same-rate condition was presented first, and the two different-tempo conditions followed. There were no differences due to the two final positions (for the different-tempo conditions) within a block. For the two positions, the average correct ratings were 1.7 $(81 \%)$ and $1.7(82 \%)$, respectively, for the 13 identicalrhythm pairs and $2.7(57 \%)$ and $2.6(56 \%)$, respectively, for the $\mathbf{2 0}$ different-rhythm pairs. Therefore, there was no evidence either for learning or for fatigue effects.

The second step was to determine whether the direction of the tempo change for different-rate presentation or whether the tempo for the same-rate presentation affected discrimination. Since the results for the identicalrhythm pairs can differ from those for the different-rhythm pairs, each will be considered separately. For the identical-rhythm pairs, there were no differences due to the direction of the tempo change. The average ratings (percent correct) across the 13 identical-rhythm pairs were $1.36(89 \%)$ for slow-medium, $1.4(90 \%)$ for medium- slow, $1.75(78 \%)$ for medium-fast, $1.73(79 \%)$ for fastmedium, and, finally, $1.83(74 \%)$ for slow-fast and 1.84 (74\%) for fast-slow. The results for the same-rate conditions were more complex. There were significant differences among the three tempos, whether with subject $[F(2,98)=5.1, p<.01]$ or with pattern $[F(2,36)=$ $5.43, p<.025]$ as the sampling variable. (Note that all of the reported analyses are based on the average ratings; analyses based on the percent correct yield identical results.) Post hoc comparisons indicated that the medium tempo was easier than the fast or the slow rate. The absolute differences were not large, however: the average ratings (percent correct) were $1.4(89 \%), 1.2(96 \%)$, and $1.4(90 \%)$ for the slow, medium, and fast rates. Given the fact that the differences among the same-rate conditions were small and that there were no differences among the pairs of different-tempo conditions, the results for the identical-rhythm pairs were combined into four tempo conditions: (1) same-rate, averaging slow, medium, and fast; (2) slow/medium, averaging slow-medium and medium-slow; (3) medium/fast, averaging medium-fast and fast-medium; and (4) slow/fast, averaging slow-fast and fast-slow.

The results for the different-rhythm pairs were similar. The differences in average rating (percent correct) between medium-fast and fast-medium were inconsequential, $2.72(58 \%)$ and $2.67(58 \%)$, and the same outcome occurred for the differences between slow-fast and fast-slow, $2.54(51 \%)$ and $2.47(49 \%)$. The differences between slow-medium and medium-slow were larger, $2.67(58 \%)$ and $2.95(66 \%)$, but this seemed due to subject variability because there was no significant difference when subjects were the sampling variable $[F(1,53)=$ $2.22, p>.10]$. Finally, there were no differences among the same-rate conditions. The average ratings (percent correct) for the slow, medium, and fast conditions were $3.19(75 \%), 3.11(72 \%)$, and $2.95(67 \%)$. On this basis, the results for the different-rhythm pairs were combined into four tempo conditions: (1) same-rate, averaging slow, medium, and fast; (2) slow/medium, averaging slowmedium and medium-slow; (3) medium/fast, averaging medium-fast and fast-medium; and (4) slow/fast, averaging slow-fast and fast-slow. Thus, the combination of the rate conditions into subgroups based on the empirical results was the same for both identical- and different-rhythm pairs.

In what follows, the results from the identical-rhythm pairs and the different-rhythm pairs will be described separately. For each kind of rhythm pair, the particular pairs will be organized on the basis of average ratings and percent correct to discover whether there was any interaction between rhythmic structure and tempo conditions.

Identical-rhythm pairs. The results for the 13 identical-rhythm pairs are shown in Table 1 for each set of presentation conditions. Here, lower average ratings indicate that subjects were able to judge correctly that the 
two rhythms were identical. The data were initially analyzed in a 4 (tempo condition) $\times 13$ (pattern) analysis of variance (ANOVA). There was a significant main effect of presentation condition $[F(3,36)=44.2, p<.001]$ and pattern $[F(12,36)=2.3, p<.05]$. Subsequent individual comparison tests (Tukey's honestly significant differences test, $p<.01$ ) indicated that there were no differences between the same-rate conditions and the slow/ medium conditions, and that the slow/fast conditions were marginally more difficult $(p<.05)$ than the medium/fast conditions. There were large differences, however, between the two more difficult conditions, slow/fast and medium/fast, and the two easier conditions, slow/medium and same rate. For the identical-rhythm pairs, the poorest performance occurred when the two rhythms were presented at different rates, and particularly when one of the rates was fast. It is not that the fast rate was more difficult in itself, because the same-rate condition with the faster rate was not more difficult than the slower rates.

The 13 patterns are presented in roughly the rank order of difficulty based on performance in the medium/fast and slow/fast conditions. This was done because the differences among patterns for the same-rate and slow-medium conditions were small. Overall, there does not seem to be a coherent interpretation of discrimination performance. The rhythm pairs seemed to split into four groups: (1) The first four rhythm pairs tended to be the hardest for both the slow/fast and the medium/fast conditions.

(2) The next five pairs were more inconsistent: Pairs 5 and 6 were relatively easy for the slow/fast conditions but relatively harder for the medium/fast conditions. (3) The reverse was true for Pairs 7, 8, and 9; these were relatively harder for the slow/fast conditions and relatively easier for the medium/fast conditions. (4) The last four pairs were easy for all presentation conditions, and particularly for the hardest medium/fast and slow/fast conditions.

There is no clear-cut way of differentiating the pattern pairs in each group. There was a tendency for rhythm pairs with a run of three tones (Pairs 6, 9, 12, and 13) to be more accurately identified as being identical. Otherwise, the results varied. For example, even patterns with the identical figural grouping (e.g., Pattern 4, x.x...x.x..x...., and Pattern 5 x.x..x.x...x...., with figural descriptions of 2-2-1) yielded very different discrimination performance in the slow/fast condition.

Different-rhythm pairs. The results for the 20 different pairs are also shown in Table 1. Here, higher average ratings indicate that subjects were able to judge correctly that the two rhythms were different. The data were initially analyzed in a 4 (presentation condition) $\times 20$ (pattern) ANOVA. There was a significant main effect for presentation condition $[F(3,57)=23.5, p<.001]$ and for pattern $[F(19,57)=17.4, p<.001]$. Subsequent individual comparison tests (Tukey's honestly significant differences test, $p<.01$ ) indicated that the same-rate con- ditions produced the best performance; the slow/medium and medium/fast conditions did not differ and produced poorer performance than did the same-rate conditions; and the slow/fast conditions produced the poorest performance of all. These results therefore suggest simply that bigger rate differences produce poorer discrimination.

The different-rhythm pairs were placed into six groups on the basis of the figural organization of each rhythm. This grouping seemed best to capture the differences in discrimination among the rhythm pairs. In what follows, the groupings are discussed in the rank order of difficulty.

The first group consisted of the three pairs in which both rhythms had identical figural organizations. For all three, there was no difference between the same-rate and the different-rate conditions, and discrimination was well below $50 \%$ correct for all presentation conditions. Perceptually, tones separated by one silent space were heard as forming groups and tones separated by two or three silent spaces were heard as being in different groups. Listeners treated all of the silent intervals between groups $(\mathrm{x} . . \mathrm{x}$ and $\mathrm{x} \ldots \mathrm{x})$ as equal. They were unable to make use of the differences in timing between the rhythmic groups, even though the $3: 4$ ratio between these onset intervals was way beyond the estimated difference threshold of $10 \%$ (Hirsh, Monahan, Grant, \& Singh, 1990). These results replicate those of Handel (1992), in which pairs of different rhythms with identical figural groupings could not be distinguished apart.

The second group consisted of eight pairs in which one rhythm had two groups or runs of two elements (i.e., two elements separated by one silent unit) while the other rhythm had one run of two elements. In all but one case (Pair 20), one run of two elements was identical for both rhythms. Corresponding to the second run in one rhythm, the other rhythm had two elements separated by two silent units (instead of the single silent unit separating two tones within a run). These patterns tended to be harder, particularly the first four (Pairs 17-20). It seems probable that these pairs of rhythms were judged as being identical because the alternation in rate disrupted temporal expectancies determining within- and between-group timings, and led to the incorrect judgments. In terms of the actual intervals, between-group timings at the fast rate (264 msec) became within-group timings at the medium $(266 \mathrm{msec})$ and slow $(167 \mathrm{msec})$ rates and between-group timings at the medium rate $(399 \mathrm{msec})$ became withingroup timings at the slow rate $(334 \mathrm{msec})$.

The third group consisted of two rhythm pairs. For each pair, one rhythm contained one group of three elements (x.x.x), the other rhythm had one group of two elements, and the group of three and two overlapped in the sequence. These rhythms were often judged as being identical, particularly for the slow/fast presentations. This outcome may be understood in the same fashion as for the second group. The rate change led subjects to hear incorrectly $x . x . . x$ or X..X.X as X.X.X or led subjects to hear incorrectly $\mathrm{X} . \mathrm{X} . \mathrm{X}$ 
as $\mathrm{x} . \mathrm{X} . \mathrm{x}$ or $\mathrm{x} . \mathrm{x} . \mathrm{x}$. Subjects heard the 2-1 figural groupings as one run of three elements or vice versa.

The fourth group consisted of three pairs in which each rhythm had one run of two elements and the runs did not overlap. Discrimination was fairly good for these rhythm pairs, probably because rate changes would not change the figural grouping.

The fifth group consisted of three rhythm pairs in which one pattern had a group of three tones and the other rhythm had two groups of two elements. The three rhythm pairs produced different levels of performance. Rhythm Pair 30 yielded poor performance. Looking at the representation in Table 1, the run of three elements in No. 12 overlaps the sequence $x . x . . x$ in No. 7. As argued previously, the rate change can lead subjects to hear $x . x . . x$ in No. 7 as being equal to $x . x . x$ in No. 12. In contrast, No. 31 produced relatively good discrimination and No. 32 produced excellent discrimination. For each of these pairs, the two groups of two in the one rhythm were adjacent so that even when the tempo changed, subjects continued to hear the rhythm figurally as $2-2-1$ (No. 5) or 1-2-2 (No. 10) and therefore were able to discriminate it from the 1-3-1 figural grouping of No. 6. Rhythm Pair 32 was easier than Rhythm Pair 31, which seems due to the greater temporal interval between the groups of two that would preclude confusing them with a group of three.

The sixth group consisted of the easiest rhythm pair (33) in which each rhythm contained a group of three tones (1-3-1 vs. $3-1-1)$. This outcome is presumably due to the fact that each group of three maintained its coherence across rate changes, which thereby allowed subjects to perceive the rhythms as being different.

An alternative conceptualization may be made in terms of a possible pattern $\times$ tempo condition interaction. Although it is impossible to test this effect statistically, the outcomes suggest that the differences between pattern conditions changed as a function of tempo condition. For the first group of rhythm pairs, in which the two different rhythms had the same figural organization, performance was identical for all conditions. However, for the second group of eight, the same-rate conditions yielded the best discrimination, the slow/medium and medium/fast conditions were intermediate, and the slow/fast condition was worst. For the next three groups (Pairs 25-32), performance on the slow/medium condition improved to the degree that it equaled that on the same-rate conditions, although performance on medium/fast and slow/fast conditions improved only slightly. For the easiest rhythm pair (33), performance was again equivalent on all tempo conditions. Thus, differences between tempo conditions occurred for the rhythm pairs in which rate changes were likely to alter the figural grouping.

To summarize these results, two rhythms were perceived as identical to the extent that rate changes led to a confusion in the figural grouping. Thus, $x . . x . . x$ might be heard as $x . x \ldots x$ or $x . . x . x$ could be heard as $x . x . x$. The change in rate undermines the expectancies among timings within and between groups of tones built up at one rate and introduces uncertainties about the correct grouping.

\section{Discussion}

It is clear that changes in rate affect the ability to discriminate timing differences between two rhythms. As the degree of tempo change increased, discrimination became progressively more difficult. The range of rate changes used here was too limited to create a function relating the percent change to the decrement in performance. Moreover, it is also premature to speculate whether the identical percent change in rate would have the same effects at different initial tempos (i.e., 1 tone/ $\mathrm{sec}$ to 1.5 tones/sec as opposed to 3 tones/sec to 4.5 tones/sec).

The changes in rate appear to disrupt the anticipations underlying the placing of tones into groups. When one is listening to a trial, there is a sense of picking up the timing and grouping by the end of the initial repetition of the first pattern. Then, when the second pattern begins at a different rate, the first tones are often misgrouped. For example, at a fast- to slow-rate change, the first tones at the slow rate if separated by one silent unit (x.x equals an onset-to-onset interval of $334 \mathrm{msec}$ at the slow rate) would first be heard as being in different successive groups, as if the fast rate were continued. But, at the slow rate, this onset interval occurs between tones within one group. Thus, subjects comment that there is essentially a figure-ground reversal as the second rate begins to generate a new set of timing expectations. The first two elements that were initially heard as forming different groups based on an extrapolation of the fast rate now become heard as forming one group based on the timing at the slow rate. The reverse effect would occur if the rate changed from slow to fast. Tones heard as forming one group at the slow rate (onset-to-onset intervals of $334 \mathrm{msec}$ ) are between-group intervals at the fast rate and fall at the midpoint of between- and within-group timings at the medium rate.

The results from the different-rhythm pairs support these arguments. The different-rhythm pairs that were most likely to be judged as being identical were either those in which the two rhythms had the identical figural grouping or those in which the timing differences could be masked by the change in rate. It is useful to visualize the $x$, notation in Table 1. Exempting the three pairs with the same figural group, the four hardest patterns (Pairs 17-20) occur in the second group. As the rate speeds or slows, we can visualize the representation as contracting or expanding in length. It is easy to see how one rhythm then comes to resemble the other more closely. For example, consider Pair 20. If the second (bottom) rhythm is presented at a faster rate (i.e., visually contracted) or if the first (top) rhythm is presented at a slower rate (i.e., visually expanded), the two rhythms come to resemble each other more closely. The different-rhythm pairs that 
were easy to discriminate were those in which the timings and groupings did not come into closer correspondence as the rate changed (e.g., Rhythm Pairs 29 and 32). It should be noted that difficulty in the same-rate conditions does not necessarily correlate with difficulty in the different-tempo-rate conditions. For example, in the samerate conditions, Rhythm Pairs 21 and 26 were quite easy to discriminate, but in the slow/fast conditions, subjects often judged the two rhythms as being the same. With both of these pairs, changing the rate could trick subjects into perceiving the same figural grouping incorrectly.

Relative and absolute organization. These results demonstrate that rhythmic organization is both relative and absolute. It is relative in the sense that it is possible to change the tempo and still perceive the identical organization. The degree to which the rate can change without affecting the organization will be determined by the type of rhythm and the original tempo. It is probable that the organization of simple, metric rhythms may be invariant over a wider range of rates. For example, Marshburn and Jones (1985) constructed 4 simple two- and three-note rhythms based on note durations (e.g., a three-note sequence of 440,440 , and $880 \mathrm{msec}$ ) and initially trained subjects to label each one. They then presented the four rhythms at different note durations, and the subjects attempted to label each one correctly. On the whole, their results showed a larger degree of constancy than those found here, particularly when the rhythm was slowed. In their experiment, identification was nearly perfect even when the rate was more than two times slower. When the rhythm became faster, identification became poorer, although even at the fastest rate it was well above chance (63\% correct). This greater degree of constancy can be attributed in part to the simpler rhythms and in part to the test context.

Marshburn and Jones (1985) point out another aspect of relative perception. Performance was determined by the degree of tempo change, not by the absolute tempo. For example, going from 4 elements/sec to 1 element $/ \mathrm{sec}$ produced a greater drop in performance than did going from 2 elements/sec to 1 element/sec, even though the testing rate was the same.

Rhythmic organization is absolute in several ways. First, there seem to be upper and lower timing bounds for rhythmic organization. Rhythmic organization is unlikely if the onset-to-onset interval falls much below $200 \mathrm{msec}$ or if the onset-to-onset interval goes much above $2 \mathrm{sec}$. These limits were originally proposed by Fraisse (1963) and recently demonstrated by Warren, Gardner, Brubaker, and Bashford (1991) for melodies.

Second, rhythmic organization at slower rates (rates slower than 2 elements/sec) is different from organization at rates greater than 3-4 elements/sec. Both values are based on outcomes cited in the introduction and on the results found here. This is not to argue that two rhythms that straddle 3 elements/sec will necessarily be perceived differently. Instead, it is to argue that, in general, slower rhythms (less than 2 elements/sec) will be perceived differently from faster rhythms (greater than 4 elements $/ \mathrm{sec})$. Thus, the slow rate $(1.9$ elements $/ \mathrm{sec})$ and medium rate $(2.3$ elements/sec) would lead to one kind of organization and the fast rate $(3.6$ elements $/ \mathrm{sec})$ would lead to another kind of organization. This supposition can be tested by using the pairs of identical rhythms, because subjects would be listening to the identical relative timing at all presentation rates. The results back up this claim. Rhythmic organization ought to be the same at the slow and medium tempos because both rates are below 3 elements/sec. Thus, discrimination for the slow/medium condition should equal that for the slow and medium samerate conditions, and in fact there was no difference. Conversely, rhythmic organization at the slow- and mediumrate conditions should be different from organization at the fast-rate condition, because the fast rate is greater than 3 elements/sec. Thus, discrimination for the slow/fast and medium/fast conditions should be poorer than that for the same-rate conditions, and in fact there was a significant decrement. It is important to note that the discrimination is equivalent at the medium/fast and slow/fast conditions in spite of the large differences in percent change of rate (medium/fast equals $57 \%$, slow/fast equals $90 \%$ ) so that it is not merely the amount of rate change that determines performance. In sum, it is the shift in organization that creates the drop in discrimination and not the amount of tempo change.

Are there preferences for tempos? When I listen to these rhythms, my sense is that the rhythm is different at the slow and fast rates. The rhythms at the slow rate sound syncopated, whereas the rhythms at the fast rate seem to have different beats. To discover whether there are tempo preferences, a small experiment was run with identical-rhythm pairs presented at the three different-rate conditions. In each trial, the rhythm was presented at two different rates and subjects judged which rate made the pattern sound more rhythmical. The subjects were told that it was impossible to define "rhythmical," and that they were to use their own judgments.

Sixteen patterns were used: the 13 patterns shown in Table 1 plus the 3 patterns beginning with an element followed by three silent units (i.e., x...x..x.x.x...., x...x.x..x.x...., and X...x.x.x..x....). All 16 possible patterns were used, because there were no time constraints in the experiments. Each pattern was presented at the six possible rate-change conditions: slow-medium; mediumslow; medium-fast; fast-medium; slow-fast; fast-slow. This generated a total of 96 conditions. Each subject was presented one half or 48 conditions to avoid fatigue and boredom. Within each group of 48 , each pattern was presented three times, once at either slow-medium or medium-slow, once at either medium-fast or fast-medium, and once at either slow-fast or fast-slow. For each trial, a rhythm was repeated four times at one rate and then 
four times at a different rate. At both rates, the tone frequency was a $440-\mathrm{Hz}$ triangle wave to avoid choice based on a tonal preference. The conditions within each block were varied across subjects, and the orders of presentation of the conditions within each block were counterbalanced across subjects. There were 40 subjects.

There were large and consistent differences among the subjects. The first group of 23 subjects consistently preferred the faster rate for all patterns; the percentages of choices were $17 \% / 83 \%$ for slow/medium, $24 \% / 76 \%$ for medium/fast, and $13 \% / 87 \%$ for slow/fast. The second group of 11 subjects preferred the medium and fast rates; the percentages of choices were $22 \% / 78 \%$ for slow/ medium, $54 \% / 64 \%$ for medium/fast, and $39 \% / 61 \%$ for slow/fast. The third group of 6 subjects preferred the slower rate; the percentages of choices were $61 \% / 39 \%$ for slow/medium, $75 \% / 25 \%$ for medium/fast, and $79 \% / 21 \%$ for slow/fast. The choices for the medium- and slow-rate preference groups were more variable than those for the fast-rate preference group, in that the preferred rate would reverse for some patterns.

Thus, there were distinct rate preferences for the faster tempos that cut across all rhythms. Although, as stated above, there were some reversals for the medium- and slow-rate preference subjects, there did not seem to be any consistent specific figural grouping or timing characteristic that generated those reversals. Unfortunately, because there was no interaction between the different rhythms and different rates, the results do not help explain whether the degree of rhythm constancy across tempos was a function of the timing and figural grouping of individual rhythms.

In summary, the degree of rhythm organization constancy across tempo or rate is a function of the type of rhythm. With simple metric rhythms, constancy will probably extend over a wide range of tempos and begin to break down only at rates that approach perceptual or memory limitations. With complex nonmetric rhythms, constancy will extend only over a narrow range of tempos, will be a function of the specific timings of each rhythm, and will reflect different organizational principles that may occur at slower and faster rates. (Obviously, constancy will also be affected by a wide range of other variables, such as subject experience, methodology, and experimental context.)

\section{EXPERIMENT 2}

The purpose of Experiment 2 was to investigate the effect of element duration on rhythm constancy. The tone durations were varied within the identical onset-to-onset timing to determine whether durational change would affect the listeners' ability to discriminate between two rhythms. The strategy was analogous to that in Experiment 1 . In the baseline conditions, the two rhythms were presented with the same tonal durations, either shorter or longer. In the experimental conditions, one rhythm was presented with shorter duration elements and the other rhythm was presented with longer duration elements. Through comparison of discrimination in the two types of condition, it was possible to determine whether duration per se affected rhythm organization.

\section{Method}

Subjects. All 67 subjects were undergraduate volunteers at the University of Tennessee who received course credit for their participation. The subjects were run in small groups of one to three.

Rhythms. All rhythms, with one exception, were the same as those in Experiment 1 (see Table 1). The 13 identical-rhythm pairs were the same as those used in Experiment 1. The different-rhythm pairs were a slightly different set from that used in Experiment 1. Of the 23 different-rhythm pairs used here, 18 were also used in Experiment 1 . The 5 new ones were chosen to include some other pairs and to include another pair with the identical figural organization (e.g., $x \ldots . x . x . x . . x \ldots$ and $x . . x . x . x . . x \ldots .$. in which the figural organization of each rhythm is 1-3-1). It should be noted that the first rhythm, x...x.x.x..x...., was used only for this pair.

The pairs were selected to create a variety of differences in the same fashion as in Experiment 1. Across the 23 different pairs, each rhythm was used three or more times (except for No. 9, which was used (wice), and each rhythm occurred equally often as the first and second rhythms of the pair. Across the conditions, the first and second rhythms of each pair were reversed to balance any order effects.

Task. The task was identical to that in Experiment 1. On every trial, two rhythms were presented, and subjects judged whether the rhythms were the same or different in terms of the timing among the elements. The subjects used a four-point rating scale, in which 1 represented very sure identical, 2 represented fairly sure identical, 3 represented fairly sure different, and 4 represented very sure different. The first rhythm was always presented with a lower pitch tone $(400 \mathrm{~Hz}$, triangle waveform) and the second rhythm was always presented with a higher pitch tone $(600 \mathrm{~Hz}$, triangle waveform).

Presentation rates. The rhythms were presented at two of the three rates used in Experiment 1. At the slow rate, the onset-toonset interval between adjacent units was $167 \mathrm{msec}$, so that there were 1.9 elements/sec. At the faster rate, the onset-to-onset interval was $88 \mathrm{msec}$, so that there were 3.6 elements $/ \mathrm{sec}$.

Tonal duration. There were 10 duration conditions. In 8 conditions, the long-duration tone was twice as long as the short-duration tone. At the fast rate, the short-duration tone was $50 \mathrm{msec}$ and the long-duration tone was $100 \mathrm{msec}$. At the slow rate, the shortduration tone was $100 \mathrm{msec}$ and the long-duration tone was $200 \mathrm{msec}$. In two conditions, the duration of the longer tones was increased to the point at which the separation between two tones within a group was just clearly noticeable. The duration at the fast rate was $140 \mathrm{msec}$, and the duration at the slow rate was $280 \mathrm{msec}$. These were termed the extended-duration conditions. For all tones, the initial and final $10 \mathrm{msec}$ consisted of an onset and offset ramp to minimize transients.

Duration conditions. For all conditions, the first rhythm was repeated twice, and then, without a pause, the second was repeated twice. Preliminary work had indicated that discriminating these rhythms was not difficult, so it was thought that the single alternation between rhythm sequences would generate more errors because it was one of the more difficult conditions in Handel (1992).

The 10 conditions can be logically split into two major parts. In the first part, the tones of the first and second rhythms had the same duration. These were six conditions, three at the slow and three 
at the fast rate. At each rate, the tones could be short, long, or extended. In the second part, the tones of the first and second rhythms had different durations. These were four conditions. At both the slow and fast rates, there were two conditions: short-long and longshort.

Experimental design. The 10 conditions were broken into 2 blocks of 5 conditions in two separate ways. In the first, the 1 st block consisted of the short-long and long-long conditions at the slow rate and the long-short, short-short, and extended-extended conditions at the fast rate, whereas the 2 nd block consisted of the remaining 5 conditions. Thus, the conditions within a block were balanced across all experimental variables. In the second, the 1st block consisted of the short-long, long-short, and extended-extended at the slow rate and the short-short and long-long at the fast rate, whereas the 2 nd block consisted of the remaining 5 conditions. Again, the conditions within a block were balanced across the experimental variables. Thus, this procedure generated 4 different blocks. The order of presentation within each of the 4 blocks was then counterbalanced to produce four different orders. Taken together, this procedure yielded 16 experimental blocks.

Each subject was presented one block of five conditions. Each block consisted of 36 rhythm pairs -13 identical pairs and 23 different pairs presented in random order (the 1 st rhythm pair was repeated later among the 36 experimental trials, so that there were 37 trials per condition). As in Experiment 1, before the actual presentation, four practice trials with simpler four-tone rhythms were given. The subjects did not receive any feedback about their performance. There were short breaks between the five conditions, so that the experimental session lasted between 75 and $90 \mathrm{~min}$. The presentation conditions were identical to those in Experiment 1 . The number of subjects for each condition was either 33 or 34 .

\section{Results}

As in Experiment 1, the data were summarized by using the average ratings and the percent correct was obtained by combining the 1 rating (very sure identical) and 2 rating (fairly sure identical) for the identical pairs and combining the 3 rating (fairly sure different) and 4 rating (very sure different) for the different pairs.

Order of presentation. Preliminary analyses indicated that there were no differences due to the order of presentation. For the five positions, the average ratings (percent correct) were $1.5(88 \%), 1.4(92 \%), 1.5(90 \%)$, 1.4.(94\%), and $1.4(91 \%)$ for the identical-rhythm pairs and $2.8(59 \%), 2.8(61 \%), 2.7(57 \%), 2.8(59 \%)$, and $2.7(58 \%)$ for the different-rhythm pairs. Given that there were no systematic changes, the data were averaged across the five positions.

As in Experiment 1, the following explanation of the results will address, first, the identical-rhythm pairs, and next, the different-rhythm pairs.

Identical-rhythm pairs. The first step in the analysis was to determine whether there were any differences among the three same-duration conditions (e.g., shortshort, long-long, extended-extended) and the two differentduration conditions (short-long, long-short) at either the fast or the slow rate. The results indicated that the differences between similar conditions were small and nonsignificant. For the short-short, long-long, and extendedextended conditions, the average ratings (percent correct) were $1.5(88 \%), 1.4(90 \%)$, and $1.5(92 \%)$, respectively,
Table 2

Average Ratings (AR) and Percent Correct (PC) for Rhythm Pairs at Slow and Fast Presentation Rates and in Same-Duration and Different-Duration Conditions

\begin{tabular}{lcccccc}
\hline & & \multicolumn{4}{c}{ Duration } \\
\cline { 3 - 4 } Rhythm & Presentation & Same & & \multicolumn{2}{c}{ Different } \\
\cline { 3 - 4 } \cline { 6 - 8 } Pairs & Rate & AR & PC & & AR & PC \\
\hline Identical & slow & 1.47 & 90 & & 1.58 & 88 \\
Identical & fast & 1.33 & 94 & & 1.37 & 93 \\
Different & slow & 2.83 & 61 & & 2.74 & 58 \\
Different & fast & 2.74 & 58 & & 2.68 & 57 \\
\hline
\end{tabular}

at the slow rate and $1.4(94 \%), 1.4(94 \%)$, and $1.3(95 \%)$, respectively, at the fast rate. For the short-long and longshort conditions, the average ratings (percent correct) were $1.6(88 \%)$ and $1.6(93 \%)$ at the slow rate and 1.4 $(92 \%)$ and $1.4(92 \%)$ at the fast rate. On this basis, the results were combined for equivalent conditions.

The results (i.e., average rating and percent correct) combined across the 13 identical rhythm pairs are shown in Table 2 for each set of duration conditions and presentation rates. Here, the fast presentation rate yielded significantly better performance than did the slow presentation rate, for both the same-duration pairs $[F(1,12)=$ $25.4, p<.001]$ and the different-duration pairs $[F(1,12)=$ $85.7, p<.001]$. At the slow rate, the same-duration conditions produced significantly better average ratings $[F(1,12)=16.8, p<.01]$ but not percent correct than did the different-duration conditions. That the fast presentation rate yielded better performance is surprising, because there was no difference between the two rates in Experiment 1 or in Handel (1992). Most probably, this difference is due to chance variation. That the discrimination in the same-duration condition was slightly better than that in the different-duration condition at the slow rate is some evidence that the duration of tonal elements can affect rhythmic organization.

Different-rhythm pairs. As was found for the identicalrhythm pairs, there were no differences among the sameduration conditions or among the different-duration conditions at either the slow or the fast rate. For the sameduration conditions, the average ratings (percent correct) for the short-short, long-long, and extended-extended conditions were $2.8(60 \%), 2.9(63 \%)$, and $2.9(61 \%)$, respectively, at the slow rate, and $2.7(59 \%), 2.6(54 \%)$, and $2.8(62 \%)$ at the fast rate. For the different-duration conditions, the average ratings (percent correct) for the short-long and long-short conditions were $2.8(59 \%)$ and $2.7(58 \%)$ at the slow rate, and $2.6(57 \%)$ and $2.7(58 \%)$ at the fast rate. On this basis, the results were combined across the equivalent conditions.

The results, combined across the 23 different rhythm pairs, are shown in Table 2 . There were no important differences among the conditions. The slow rate produced slightly better discrimination, and within each rate, the same-duration conditions produced slightly better discrim- 
ination than did the different-duration conditions. Nonetheless, all these differences were small and none were significant.

On the whole, tonal duration did not affect discrimination to any marked degree. Although the same-duration conditions were slightly better than the different-duration conditions for both presentation rates and type of rhythm pairs, each of these differences was quite small.

\section{Discussion}

The results of this experiment support the contention that duration does not affect rhythmic organization within a fixed attack-point timing. Clearly, duration will affect organization if it changes the timings between the onsets of tones, but that is taken for granted. This experiment hardly settles the matter, however, because the present task context represents just one possibility and other contexts may lead to a different conclusion.

1. The present task required a very simple response. Subjects could identify two rhythms as being different even if they could not identify which tones had "moved" or even if they could not tell whether the difference occurred in the first or second half of the pattern. Thus, a finer grained response such as circling the tones that are different, or, alternately, a playback response in which subjects tap out the rhythm might reveal effects of duration.

2. The duration variable may not have been strong enough. The majority of conditions used a duration ratio of $2: 1$, and although that is clearly discriminable, it may not have been large enough to affect the organization. Moreover, the durations of all tones within a rhythm were identical. Clearly, this is not representative even of simple music. A duration rhythm that is overlaid on the onsetto-onset timing rhythm might create a different organization. For example, the rhythm x.x..x.x...x.... might be generated with two reversed duration rhythms: long, short, long, short, long, or short, long, short, long, short. These different duration rhythms integrated with the attack-point rhythm may lead subjects to hear the identical timing rhythms as being different.

3. Finally, the use of steady-state sounds is unrepresentative of the changes in timbre that occur at longer durations in the playing of natural instruments. Thus, the duration pattern may have its effect on rhythmic organization by means of changes in the sound quality of notes.

To summarize, it seems clear that simply changing all the durations within a rhythm does not influence rhythm discrimination. Duration may influence organization and discrimination, however, by changing the note grouping because of accents induced by a duration rhythm or because of timbre changes induced by different decay functions.

\section{GENERAL DISCUSSION}

The outcomes of Experiments 1 and 2 support the notion that rhythmic organization depends on tempo but is independent of tone duration. It seems reasonable to hypothesize that discrimination is achieved at faster rates by using global templates but is achieved at slower rates by using timing expectancies or anticipations (Ten Hoopen, 1992). These different modes are probably caused by memory limitations that preclude the build-up of templates that span more than $1.5 \mathrm{sec}$ and by perceptual limitations that preclude the build-up of accurate timing expectancies if the onset-to-onset interval is less than 250-300 msec. The rhythms at slower and faster rates do sound different, and subjects display consistent preferences. However, there is little evidence here that organization depends on element duration. This is not to argue that duration cannot affect organization in a different context.

A discrimination task imposes a set of constraints on the analysis of rhythmic organization. As described previously, it is impossible to know whether different judgments are based on the detection of the correct variation between the two rhythms. Moreover, the task masks any bias toward one kind of response. It appears that subjects, if uncertain, do have a tendency to respond that two rhythms are identical. For the most difficult conditions, this tendency probably overstates discrimination of the identical-rhythm pairs and understates discrimination of the different-rhythm pairs. Any task imposes constraints both on the subject's response capabilities and on the experimenter's ability to interpret that response. What is necessary is a set of converging operations (Garner, Hake, \& Eriksen, 1956) based on rhythm and task variations.

The fact that any task imposes constraints on rhythmic organization leads to the general question of whether any single theory of rhythm interpretation is possible. Can one theory embrace metric and nonmetric rhythms, mono- and polymeter rhythms, slow and fast tempos, identification and discrimination tasks, and so on? If a single theory is possible, that theory must go beyond listing a set of tonal attributes that may contribute to organization. In such a theory, it would be expected that different organizations would emerge from the same timing structure in different contexts. The theory would be intrinsically interactional.

\section{REFERENCES}

Bamberger, J. (1978). Intuitive and formal musical knowing: Parables of cognitive dissonance. In S. S. Madeja (Ed.), The arts, cognition and basic skills (pp. 173-209). St. Louis, MO: CEMREL.

Bregman, A. S. (1990). Auditory scene analysis. Cambridge, MA: MIT Press.

Dowling, W. J. (1986). Context effects on melody recognition: Scalestep versus interval representations. Music Perception, 3, 281-296.

FraISSE, P. (1963). The psychology of time. New York: Harper \& Row.

GARNER, W. R. (1974). The processing of information and structure. Potomac, MD: Erlbaum.

Garner, W. R., Hake, H. W., \& Eriksen, C. W. (1956). Operationism and the concept of perception. Psychological Review, 63, 149-159.

HANDEL, S. (1974). Perceiving melodic and thythmic auditory patterns. Journal of Experimental Psychology, 103, 922-933.

Handel, S. (1992). The differentiation of rhythmic structure. Perception \& Psychophysics, 52, 497-507. 
Handel, S., \& Lawson, G. R. (1983). The contextual nature of rhythmic interpretation. Perception \& Psychophysics, 34, 103-120.

Hirsh, I. J., Monahan, C. B., Grant, K. W., \& Singh, P. G. (1990). Studies in auditory timing: 1 . Simple patterns. Perception \& P sychophysics, 47, 215-226.

Hopkins, R. G. (1992, February). Perceptual organization of eightelement melodic and rhythmic patterns. Paper presented at the 2nd International Conference on Music Perception and Cognition, Los Angeles.

LeRDAHL, F., \& JACKendofF, R. (1983). Generative theory of tonal music. Cambridge, MA: MIT Press.

Marshburn, E. A., \& Jones, M. R. (1985, May). Rhythm recognition as a function of rate: Relative or absolute? Paper presented at the meeting of the Midwestern Psychological Association, Chicago.

Monahan, C. B., \& Hirsh, I. J. (1990). Studies in auditory timing: 2. Rhythm patterns. Perception \& Psychophysics, 47, 227-242.

Palmer, C., \& Krumhansl, C. L. (1990). Mental representations for musical meter. Journal of Experimental Psychology: Human Perception \& Performance, 16, 728-741.
Povel, D.-J., \& Essens, P. (1985). Perception of temporal patterns. Music Perception, 2, 411-440.

Sorkin, R. D., \& Montgomery, D. A. (1991). Effect of time compression and expansion on the discrimination of tonal patterns. Joumal of the Acoustical Society of America, 90, 846-857.

Ten Hoopen, G. (1992, February). Temporal processing of fast auditory patterns. Paper presented at the 2nd International Conference on Music Perception and Cognition, Los Angeles.

Vos, P. G., \& Ellerman, H. H. (1989). Precision and accuracy in the reproduction of simple tone sequences. Journal of Experimental Psychology: Human Perception \& Performance, 15, 179-187.

Warren, R. M., Gardner, D. A., Brubaker, B. S., \& Bashford, JR., J. A. (1991). Melodic and nonmelodic sequences of tones: Effects of duration on perception. Music Perception, 8, 277-289.

Yeston, M. (1976). The stratification of musical rhythm. New Haven: Yale University Press.

(Manuscript received September 3, 1992; revision accepted for publication February 17, 1993.) 